\title{
Correction to: Economic Self-Interest and Americans' Redistributive, Class, and Racial Attitudes: The Case of Economic Insecurity
}

\section{Cody R. Melcher ${ }^{1}$ (D)}

Published online: 13 April 2021

(c) Springer Science+Business Media, LLC, part of Springer Nature 2021

\section{Correction to: Political Behavior https://doi.org/10.1007/s11109-021-09694-x}

The original version of this article unfortunately contained a mistake in Tables 1,2 and 3. The Tables continued page contains the incorrect column headers. The corrected Tables 1,2, 3 are given below.

The original article can be found online at https://doi.org/10.1007/s11109-021-09694-x.

Cody R. Melcher

cmelcher@gradcenter.cuny.edu

1 Department of Sociology, The Graduate Center at the City University of New York, New York, NY, USA 
Table 1 Economic insecurity and redistributive attitudes

A. Government should reduce income differences (Disagree strongly to agree strongly; 5 categories)

\begin{tabular}{|c|c|c|c|c|c|}
\hline Past worse & $\begin{array}{l}0.139 \\
(0.120)\end{array}$ & & & & $\begin{array}{l}0.153 \\
(0.152)\end{array}$ \\
\hline Future worse & & $\begin{array}{l}-0.249 * \\
(0.136)\end{array}$ & & & $\begin{array}{l}-0.293 * \\
(0.166)\end{array}$ \\
\hline Job insecure & & & $\begin{array}{l}0.223^{*} \\
(0.129)\end{array}$ & & $\begin{array}{l}0.173 \\
(0.140)\end{array}$ \\
\hline Financial anxiety & & & & $\begin{array}{l}0.251 * * * \\
(0.095)\end{array}$ & $\begin{array}{l}0.178 \\
(0.138)\end{array}$ \\
\hline National economy & $\begin{array}{l}-0.015 \\
(0.127)\end{array}$ & $\begin{array}{l}0.088 \\
(0.126)\end{array}$ & $\begin{array}{l}0.027 \\
(0.152)\end{array}$ & $\begin{array}{l}0.011 \\
(0.122)\end{array}$ & $\begin{array}{l}0.017 \\
(0.164)\end{array}$ \\
\hline Ideology & $\begin{array}{l}-1.249 * * * \\
(0.165)\end{array}$ & $\begin{array}{l}-1.276^{* * *} \\
(0.165)\end{array}$ & $\begin{array}{l}-1.127 * * * \\
(0.194)\end{array}$ & $\begin{array}{l}-1.240 * * * \\
(0.170)\end{array}$ & $\begin{array}{l}-1.100^{* * * *} \\
(0.197)\end{array}$ \\
\hline Party ID & $\begin{array}{l}-0.720^{* * * *} \\
(0.110)\end{array}$ & $\begin{array}{l}-0.687 * * * \\
(0.108)\end{array}$ & $\begin{array}{l}-0.783^{* * * *} \\
(0.139)\end{array}$ & $\begin{array}{l}-0.699 * * * \\
(0.111)\end{array}$ & $\begin{array}{l}-0.790^{* * * *} \\
(0.139)\end{array}$ \\
\hline Racial resentment & $\begin{array}{l}-0.890 * * * \\
(0.109)\end{array}$ & $\begin{array}{l}-0.902 * * * \\
(0.109)\end{array}$ & $\begin{array}{l}-1.098 * * * \\
(0.149)\end{array}$ & $\begin{array}{l}-0.913 * * * \\
(0.111)\end{array}$ & $\begin{array}{l}-1.110^{* * * *} \\
(0.150)\end{array}$ \\
\hline Constant & $\begin{array}{l}5.058 * * * \\
(0.164)\end{array}$ & $\begin{array}{l}5.151 * * * \\
(0.155)\end{array}$ & $\begin{array}{l}5.189 * * * \\
(0.198)\end{array}$ & $\begin{array}{l}4.965 * * * \\
(0.166)\end{array}$ & $\begin{array}{l}5.151 * * * \\
(0.208)\end{array}$ \\
\hline $\mathrm{N}$ & 2639 & 2624 & 1773 & 2643 & 1761 \\
\hline Log likelihood & -4287.739 & -4257.384 & -2848.719 & -4291.214 & -2824.694 \\
\hline
\end{tabular}

B. Government should provide jobs and income (Should let each person get ahead on own; 7 categories)

\begin{tabular}{|c|c|c|c|c|c|}
\hline Past worse & $\begin{array}{l}0.216 \\
(0.154)\end{array}$ & & & & $\begin{array}{l}0.098 \\
(0.209)\end{array}$ \\
\hline Future worse & & $\begin{array}{l}0.284 \\
(0.175)\end{array}$ & & & $\begin{array}{l}0.337 \\
(0.221)\end{array}$ \\
\hline Job insecure & & & $\begin{array}{l}0.236 \\
(0.218)\end{array}$ & & $\begin{array}{l}0.070 \\
(0.221)\end{array}$ \\
\hline Financial anxiety & & & & $\begin{array}{l}0.382 * * \\
(0.147)\end{array}$ & $\begin{array}{l}0.329 * \\
(0.193)\end{array}$ \\
\hline National economy & $\begin{array}{l}0.435 * * \\
(0.178)\end{array}$ & $\begin{array}{l}0.433 * * \\
(0.180)\end{array}$ & $\begin{array}{l}0.399 * \\
(0.230)\end{array}$ & $\begin{array}{l}0.447 * * \\
(0.179)\end{array}$ & $\begin{array}{l}0.261 \\
(0.225)\end{array}$ \\
\hline Ideology & $\begin{array}{l}-1.347 * * * \\
(0.200)\end{array}$ & $\begin{array}{l}-1.304 * * * \\
(0.200)\end{array}$ & $\begin{array}{l}-1.272^{* * * *} \\
(0.269)\end{array}$ & $\begin{array}{l}-1.308 * * * \\
(0.198)\end{array}$ & $\begin{array}{l}-1.214 * * * \\
(0.264)\end{array}$ \\
\hline Party ID & $\begin{array}{l}-1.240 * * * \\
(0.164)\end{array}$ & $\begin{array}{l}-1.257 * * * \\
(0.163)\end{array}$ & $\begin{array}{l}-1.429 * * * \\
(0.203)\end{array}$ & $\begin{array}{l}-1.219 * * * \\
(0.164)\end{array}$ & $\begin{array}{l}-1.433^{* * * *} \\
(0.204)\end{array}$ \\
\hline Racial resentment & $\begin{array}{l}-1.307 * * * \\
(0.171)\end{array}$ & $\begin{array}{l}-1.297 * * * \\
(0.171)\end{array}$ & $\begin{array}{l}-1.419 * * * \\
(0.196)\end{array}$ & $\begin{array}{l}-1.344 * * * \\
(0.170)\end{array}$ & $\begin{array}{l}-1.450 * * * \\
(0.199)\end{array}$ \\
\hline Constant & $\begin{array}{l}5.749 * * * \\
(0.189)\end{array}$ & $\begin{array}{l}5.722 * * * \\
(0.197)\end{array}$ & $\begin{array}{l}5.881 * * * \\
(0.215)\end{array}$ & $\begin{array}{l}5.630^{* * * *} \\
(0.192)\end{array}$ & $\begin{array}{l}5.660 * * * \\
(0.245)\end{array}$ \\
\hline $\mathrm{N}$ & 2481 & 2466 & 1673 & 2484 & 1661 \\
\hline Log likelihood & -4708.169 & -4679.224 & -3139.532 & -4708.193 & -3112.322 \\
\hline & \multicolumn{5}{|c|}{ C. How much opportunity to get ahead? (A great deal to None; 5 categories) } \\
\hline Past worse & $\begin{array}{l}0.739 * * * \\
(0.095)\end{array}$ & & & & $\begin{array}{l}0.296 * * \\
(0.138)\end{array}$ \\
\hline
\end{tabular}


Table 1 (continued)

C. How much opportunity to get ahead? (A great deal to None; 5 categories)

\begin{tabular}{|c|c|c|c|c|c|}
\hline \multicolumn{2}{|l|}{ Future worse } & \multicolumn{3}{|l|}{$\begin{array}{l}0.571 * * * \\
(0.129)\end{array}$} & $\begin{array}{l}0.325 * * \\
(0.160)\end{array}$ \\
\hline Job insecure & & & $\begin{array}{l}0.413 * * * \\
(0.118)\end{array}$ & & $\begin{array}{l}0.062 \\
(0.118)\end{array}$ \\
\hline Financial anxiety & & & & $\begin{array}{l}0.960 * * * \\
(0.079)\end{array}$ & $\begin{array}{l}0.840 * * * \\
(0.101)\end{array}$ \\
\hline National economy & $\begin{array}{l}0.442 * * * \\
(0.120)\end{array}$ & $\begin{array}{l}0.539 * * * \\
(0.117)\end{array}$ & $\begin{array}{l}0.593 * * * \\
(0.141)\end{array}$ & $\begin{array}{l}0.521 * * * \\
(0.110)\end{array}$ & $\begin{array}{l}0.368 * * * \\
(0.129)\end{array}$ \\
\hline Ideology & $\begin{array}{l}-0.270 \\
(0.178)\end{array}$ & $\begin{array}{l}-0.236 \\
(0.176)\end{array}$ & $\begin{array}{l}-0.225 \\
(0.196)\end{array}$ & $\begin{array}{l}-0.154 \\
(0.167)\end{array}$ & $\begin{array}{l}-0.130 \\
(0.184)\end{array}$ \\
\hline Party ID & $\begin{array}{l}-0.219 * \\
(0.118)\end{array}$ & $\begin{array}{l}-0.226^{*} \\
(0.116)\end{array}$ & $\begin{array}{l}-0.206 \\
(0.150)\end{array}$ & $\begin{array}{l}-0.170 \\
(0.114)\end{array}$ & $\begin{array}{l}-0.229 \\
(0.143)\end{array}$ \\
\hline Racial resentment & $\begin{array}{l}-0.289 * * * \\
(0.106)\end{array}$ & $\begin{array}{l}-0.261 * * \\
(0.108)\end{array}$ & $\begin{array}{l}-0.297 * * \\
(0.132)\end{array}$ & $\begin{array}{l}-0.372 * * * \\
(0.104)\end{array}$ & $\begin{array}{l}-0.388 * * * \\
(0.127)\end{array}$ \\
\hline Constant & $\begin{array}{l}3.355^{* * * *} \\
(0.122)\end{array}$ & $\begin{array}{l}3.429 * * * \\
(0.115)\end{array}$ & $\begin{array}{l}3.525^{* * * *} \\
(0.152)\end{array}$ & $\begin{array}{l}3.111^{* * * *} \\
(0.116)\end{array}$ & $\begin{array}{l}3.138 * * * \\
(0.149)\end{array}$ \\
\hline $\mathrm{N}$ & 2648 & 2632 & 1779 & 2652 & 1766 \\
\hline Log likelihood & -3889.283 & -3875.653 & -2609.387 & -3827.411 & -2520.210 \\
\hline
\end{tabular}

Models also control for income, education level, age, union membership, gender, religiosity, and race $* \mathrm{p}<.1 ; * * \mathrm{p}<.05 ; * * * \mathrm{p}<.01 ;$ standard errors (in parentheses) corrected for cluster sampling 
Table 2 Economic insecurity and attitudes toward class actors

\begin{tabular}{|c|c|c|c|c|c|}
\hline \multirow[b]{2}{*}{ Past worse } & \multicolumn{5}{|c|}{ A. Big business $(0-100)$} \\
\hline & $\begin{array}{l}-8.146^{* * * *} \\
(2.150)\end{array}$ & & & & $\begin{array}{l}-5.099 \\
(3.136)\end{array}$ \\
\hline Future worse & & $\begin{array}{l}-9.128 * * * \\
(2.551)\end{array}$ & & & $\begin{array}{l}-6.311 * \\
(3.457)\end{array}$ \\
\hline Job insecure & & & $\begin{array}{l}-1.339 \\
(2.471)\end{array}$ & & $\begin{array}{l}1.322 \\
(2.653)\end{array}$ \\
\hline Financial anxiety & & & & $\begin{array}{l}-6.303^{* * * *} \\
(1.751)\end{array}$ & $\begin{array}{l}-5.031 * * \\
(2.170)\end{array}$ \\
\hline National economy & $\begin{array}{l}-0.141 \\
(2.494)\end{array}$ & $\begin{array}{l}-1.364 \\
(2.698)\end{array}$ & $\begin{array}{l}-4.886 \\
(3.545)\end{array}$ & $\begin{array}{l}-2.197 \\
(2.602)\end{array}$ & $\begin{array}{l}-1.774 \\
(3.651)\end{array}$ \\
\hline Ideology & $\begin{array}{l}16.970 * * * \\
(3.280)\end{array}$ & $\begin{array}{l}17.098 * * * \\
(3.384)\end{array}$ & $\begin{array}{l}17.323 * * * \\
(4.097)\end{array}$ & $\begin{array}{l}16.559 * * * \\
(3.290)\end{array}$ & $\begin{array}{l}17.046 * * * \\
(4.166)\end{array}$ \\
\hline Party ID & $\begin{array}{l}5.932 * * \\
(2.446)\end{array}$ & $\begin{array}{l}5.697 * * \\
(2.496)\end{array}$ & $\begin{array}{l}7.418^{* * *} \\
(3.288)\end{array}$ & $\begin{array}{l}5.417 * * \\
(2.497)\end{array}$ & $\begin{array}{l}7.524 * * \\
(3.355)\end{array}$ \\
\hline Racial resentment & $\begin{array}{l}12.384 * * * \\
(2.504)\end{array}$ & $\begin{array}{l}12.008 * * * \\
(2.480)\end{array}$ & $\begin{array}{l}11.485^{* * * *} \\
(3.126)\end{array}$ & $\begin{array}{l}12.889 * * * \\
(2.508)\end{array}$ & $\begin{array}{l}11.756^{* * * *} \\
(3.133)\end{array}$ \\
\hline Constant & $\begin{array}{l}26.421 * * * \\
(3.198)\end{array}$ & $\begin{array}{l}27.120 * * * \\
(3.066)\end{array}$ & $\begin{array}{l}23.429 * * * \\
(4.148)\end{array}$ & $\begin{array}{l}27.491 \text { *** } \\
(3.163)\end{array}$ & $\begin{array}{l}27.842^{* * * *} \\
(4.211)\end{array}$ \\
\hline $\mathrm{N}$ & 2630 & 2615 & 1772 & 2634 & 1759 \\
\hline \multirow[t]{2}{*}{ Log likelihood } & $-11,936.720$ & $-11,874.480$ & -8039.639 & $-11,961.300$ & -7965.974 \\
\hline & \multicolumn{5}{|c|}{ B. Rich people (0-100) } \\
\hline Past worse & $\begin{array}{l}-5.528 * * \\
(2.208)\end{array}$ & & & & $\begin{array}{l}-1.541 \\
(3.146)\end{array}$ \\
\hline Future worse & & $\begin{array}{l}-9.906 * * * \\
(2.283)\end{array}$ & & & $\begin{array}{l}-7.797 * * \\
(3.154)\end{array}$ \\
\hline Job insecure & & & $\begin{array}{l}-4.253^{*} \\
(2.307)\end{array}$ & & $\begin{array}{l}-2.745 \\
(2.573)\end{array}$ \\
\hline Financial anxiety & & & & $\begin{array}{l}-5.489 * * * \\
(1.383)\end{array}$ & $\begin{array}{l}-2.362 \\
(1.863)\end{array}$ \\
\hline National economy & $\begin{array}{l}-1.037 \\
(2.380)\end{array}$ & $\begin{array}{l}-1.059 \\
(2.259)\end{array}$ & $\begin{array}{l}-3.225 \\
(2.770)\end{array}$ & $\begin{array}{l}-2.032 \\
(2.224)\end{array}$ & $\begin{array}{l}-0.970 \\
(2.857)\end{array}$ \\
\hline Ideology & $\begin{array}{l}12.643 * * * \\
(2.917)\end{array}$ & $\begin{array}{l}12.790 * * * \\
(2.919)\end{array}$ & $\begin{array}{l}12.909 * * * \\
(3.783)\end{array}$ & $\begin{array}{l}12.142 * * * \\
(2.883)\end{array}$ & $\begin{array}{l}12.368 * * * \\
(3.727)\end{array}$ \\
\hline Party ID & $\begin{array}{l}7.939 * * * \\
(1.988)\end{array}$ & $\begin{array}{l}7.655^{* *} \\
(1.996)\end{array}$ & $\begin{array}{l}7.704 * * * \\
(2.210)\end{array}$ & $\begin{array}{l}7.599 * * * \\
(1.991)\end{array}$ & $\begin{array}{l}7.957 * * * \\
(2.213)\end{array}$ \\
\hline Racial resentment & $\begin{array}{l}4.232 * * \\
(1.772)\end{array}$ & $\begin{array}{l}4.002 * * \\
(1.765)\end{array}$ & $\begin{array}{l}5.591 * * \\
(2.350)\end{array}$ & $\begin{array}{l}4.682 * * * \\
(1.751)\end{array}$ & $\begin{array}{l}5.539 * * \\
(2.289)\end{array}$ \\
\hline Constant & $\begin{array}{l}32.501 * * * \\
(2.673)\end{array}$ & $\begin{array}{l}33.732 * * * \\
(2.470)\end{array}$ & $\begin{array}{l}29.289 * * * \\
(3.183)\end{array}$ & $\begin{array}{l}33.617 * * * \\
(2.602)\end{array}$ & $\begin{array}{l}32.506^{* * * *} \\
(3.249)\end{array}$ \\
\hline $\mathrm{N}$ & 2626 & 2611 & 1766 & 2630 & 1754 \\
\hline Log likelihood & $-11,744.270$ & $-11,666.570$ & -7867.140 & $-11,759.450$ & -7801.811 \\
\hline & \multicolumn{5}{|c|}{ C. Poor people $(0-100)$} \\
\hline Past worse & $\begin{array}{l}1.374 \\
(2.007)\end{array}$ & & & & $\begin{array}{l}-1.594 \\
(2.602)\end{array}$ \\
\hline
\end{tabular}


Table 2 (continued)

C. Poor people (0-100)

\begin{tabular}{|c|c|c|c|c|c|}
\hline \multicolumn{2}{|l|}{ Future Worse } & \multicolumn{3}{|l|}{$\begin{array}{l}-2.069 \\
(2.469)\end{array}$} & $\begin{array}{l}-2.083 \\
(3.010)\end{array}$ \\
\hline Job Insecure & & & $\begin{array}{l}-1.839 \\
(2.211)\end{array}$ & & $\begin{array}{l}-3.294 \\
(2.458)\end{array}$ \\
\hline Financial anxiety & & & & $\begin{array}{l}4.191 * * \\
(1.714)\end{array}$ & $\begin{array}{l}4.537 * * \\
(2.267)\end{array}$ \\
\hline National economy & $\begin{array}{l}-2.455 \\
(2.084)\end{array}$ & $\begin{array}{l}-1.881 \\
(2.079)\end{array}$ & $\begin{array}{l}-3.610 \\
(2.972)\end{array}$ & $\begin{array}{l}-2.922 \\
(2.033)\end{array}$ & $\begin{array}{l}-3.204 \\
(3.022)\end{array}$ \\
\hline Ideology & $\begin{array}{l}1.349 \\
(3.056)\end{array}$ & $\begin{array}{l}1.566 \\
(3.126)\end{array}$ & $\begin{array}{l}2.384 \\
(3.741)\end{array}$ & $\begin{array}{l}2.073 \\
(3.063)\end{array}$ & $\begin{array}{l}2.913 \\
(3.779)\end{array}$ \\
\hline Party ID & $\begin{array}{l}-3.358^{*} \\
(1.907)\end{array}$ & $\begin{array}{l}-3.400^{*} \\
(1.934)\end{array}$ & $\begin{array}{l}-3.791 \\
(2.353)\end{array}$ & $\begin{array}{l}-3.297 * \\
(1.898)\end{array}$ & $\begin{array}{l}-3.685 \\
(2.367)\end{array}$ \\
\hline Racial resentment & $\begin{array}{l}-11.753 * * * \\
(1.875)\end{array}$ & $\begin{array}{l}-12.012 * * * \\
(1.858)\end{array}$ & $\begin{array}{l}-12.605^{* * * *} \\
(2.339)\end{array}$ & $\begin{array}{l}-12.128 * * * \\
(1.864)\end{array}$ & $\begin{array}{l}-13.232 * * * \\
(2.294)\end{array}$ \\
\hline Constant & $\begin{array}{l}71.660 * * * \\
(2.909)\end{array}$ & $\begin{array}{l}73.065 * * * \\
(2.767)\end{array}$ & $\begin{array}{l}72.977 * * * \\
(3.442)\end{array}$ & $\begin{array}{l}70.253^{* * * *} \\
(2.720)\end{array}$ & $\begin{array}{l}72.504 * * * \\
(3.714)\end{array}$ \\
\hline $\mathrm{N}$ & 2627 & 2613 & 1768 & 2631 & 1756 \\
\hline Log likelihood & $-11,672.620$ & $-11,610.890$ & -7858.365 & $-11,685.420$ & -7802.503 \\
\hline
\end{tabular}

Models also control for income, education level, age, union membership, gender, religiosity, and race $* \mathrm{p}<.1 ; * * \mathrm{p}<.05 ; * * * \mathrm{p}<.01 ;$ standard errors (in parentheses) corrected for cluster sampling 
Table 3 Economic insecurity and racial attitudes; white respondents only

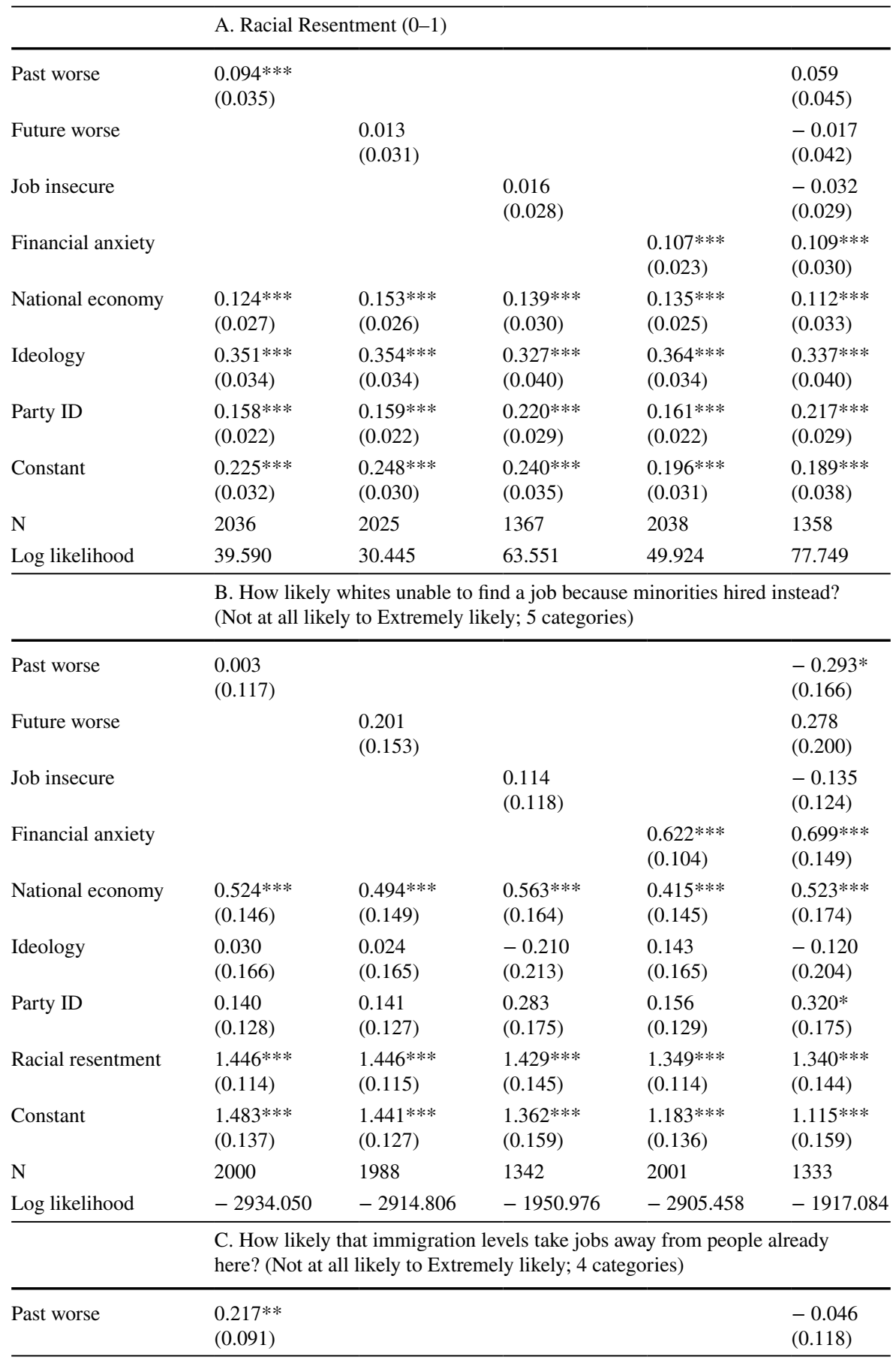


Table 3 (continued)

C. How likely that immigration levels take jobs away from people already here? (Not at all likely to Extremely likely; 4 categories)

\begin{tabular}{|c|c|c|c|c|c|}
\hline Future worse & & $\begin{array}{l}0.240 * * \\
(0.111)\end{array}$ & & & $\begin{array}{l}0.192 \\
(0.142)\end{array}$ \\
\hline Job insecure & & & $\begin{array}{l}0.259 * * \\
(0.119)\end{array}$ & & $\begin{array}{l}0.101 \\
(0.122)\end{array}$ \\
\hline Financial anxiety & & & & $\begin{array}{l}0.469 * * * \\
(0.086)\end{array}$ & $\begin{array}{l}0.381 * * * \\
(0.117)\end{array}$ \\
\hline National economy & $\begin{array}{l}0.793 * * * \\
(0.118)\end{array}$ & $\begin{array}{l}0.818 * * * \\
(0.115)\end{array}$ & $\begin{array}{l}0.742 * * * \\
(0.130)\end{array}$ & $\begin{array}{l}0.787 * * * \\
(0.114)\end{array}$ & $\begin{array}{l}0.690 * * * \\
(0.138)\end{array}$ \\
\hline Ideology & $\begin{array}{l}0.070 \\
(0.119)\end{array}$ & $\begin{array}{l}0.047 \\
(0.119)\end{array}$ & $\begin{array}{l}-0.093 \\
(0.155)\end{array}$ & $\begin{array}{l}0.137 \\
(0.119)\end{array}$ & $\begin{array}{l}-0.044 \\
(0.157)\end{array}$ \\
\hline Party ID & $\begin{array}{l}0.175^{*} \\
(0.098)\end{array}$ & $\begin{array}{l}0.184 * \\
(0.098)\end{array}$ & $\begin{array}{l}0.366^{* * * *} \\
(0.114)\end{array}$ & $\begin{array}{l}0.195 * * \\
(0.097)\end{array}$ & $\begin{array}{l}0.377 * * * \\
(0.115)\end{array}$ \\
\hline Racial Resentment & $\begin{array}{l}1.049 * * * \\
(0.109)\end{array}$ & $\begin{array}{l}1.076^{* * *} \\
(0.107)\end{array}$ & $\begin{array}{l}1.065^{* * *} \\
(0.130)\end{array}$ & $\begin{array}{l}0.993 * * * \\
(0.109)\end{array}$ & $\begin{array}{l}1.009 * * * \\
(0.131)\end{array}$ \\
\hline Constant & $\begin{array}{l}1.106 * * * \\
(0.117)\end{array}$ & $\begin{array}{l}1.108 * * * \\
(0.119)\end{array}$ & $\begin{array}{l}0.993 * * * \\
(0.119)\end{array}$ & $\begin{array}{l}0.939 * * * \\
(0.110)\end{array}$ & $\begin{array}{l}0.839 * * * \\
(0.117)\end{array}$ \\
\hline $\mathrm{N}$ & 2031 & 2020 & 1365 & 2033 & 1356 \\
\hline Log Likelihood & -2600.409 & -2580.552 & -1733.157 & -2581.815 & -1707.853 \\
\hline
\end{tabular}

Models also control for income, education level, age, union membership, gender, and religiosity $* \mathrm{p}<.1 ; * * \mathrm{p}<.05 ; * * * \mathrm{p}<.01 ;$ standard errors (in parentheses) corrected for cluster sampling

Publisher's Note Springer Nature remains neutral with regard to jurisdictional claims in published maps and institutional affiliations. 